\title{
Rounded Atelectasis Associated with Pulmonary Lymphangioleiomyomatosis
}

\author{
Kazuma Kishi, Sakae Homma, Atsushi Miyamoto, Atsuko KurosaKi*, \\ Noriko MotoI**, Tadasu KoHNO*** and Kunihiko YoshIMURA
}

\begin{abstract}
Rounded atelectasis is an unusual form of lung collapse that develops as a consequence of pleural diseases. Among a variety of conditions, asbestos inhalation has been attributed in most cases, but many other causes have also been implicated. Here, we describe the first case of rounded atelectasis and pneumothorax associated with pulmonary lymphagioleiomyomatosis.

(Internal Medicine 44: 625-627, 2005)
\end{abstract}

Key words: rounded atelectasis, pulmonary lymphangioleiomyomatosis, pneumothorax

\section{Introduction}

Rounded atelectasis is an atelectasis of the peripheral part of the lung due to pleural adhesions and fibrosis which eventually causes deformation of the lung and bending of some small bronchi. Asbestos inhalation has been attributed in most cases among a variety of conditions, but any type of pleural inflammatory reaction can result in rounded atelectasis (1). It is usually detected incidentally on chest radiograph in elderly men who are otherwise asymptomatic.

Pulmonary lymphangioleiomyomatosis (LAM) is a rare disease that occurs exclusively in women of reproductive age (2). The clinical characteristics of LAM include recurrent spontaneous pneumothorax, slowly progressive dyspnea, hemoptysis, and chylothorax. The cardinal pathological findings are proliferation of abnormal smooth muscle cells in the lung parenchyma and axial lymphatics in the thorax and abdomen, and formation of the multiple and thin-walled pulmonary cysts. Although pleural adhesions are often found in patients with LAM (3), there have been no reports of rounded atelectasis as a complication of LAM. Herein, we describe a patient who was found incidentally to have rounded atelectasis and pneumothorax associated with LAM.

\section{Case Report}

A 45-year-old woman was admitted to Toranomon Hospital for an examination of a mass and pneumothorax with a small amount of pleural effusion in the right thorax detected on mass screening by chest radiography (Fig. 1). The patient was a housewife and had been asymptomatic. Her medical record was unremarkable. She had no history of cigarette smoking or exposure to asbestos. There was no family history of tuberous sclerosis. Physical examination showed a decrease of respiratory sound on the right hemithorax. Hematologic and blood chemical tests were normal. The previous chest radiograph taken 18 months earlier showed a slight pleural thickening on the right without a mass lesion in the right lower lung field. A chest CT scan revealed multiple thin-walled cysts throughout both lungs and right-sided pneumothorax with a small amount of pleural effusion (Fig. 2). Further, a well-defined rounded mass $(4 \times 4$ $\mathrm{cm}$ in diameter) was seen in the superior segment of the right lower lobe which contained scattered calcification and was contiguous with thickened pleura. Curvilinear opacities composed of bronchi and vessels extending from the upper portion of the mass lesion toward the hilum and extensive thickening of the visceral and parietal pleura were also recognized. No abnormality suggestive of extrapulmonary lesions of LAM was found by brain and abdominal CT scans. Chest drainage was not performed for the treatment of pneumothorax.

Video-assisted thoracoscopic surgery (VATS) was performed as a diagnostic procedure on the thirteenth hospital day. Radiographic findings remained unchanged from the admission to the day of VATS. Macroscopically, adhesion

From the Department of Respiratory Medicine, Respiratory Center, *Department of Diagnostic Radiology, **Department of Pathology and ***Department of Thoracic Surgery, Toranomon Hospital, Tokyo

Received for publication August 31, 2004; Accepted for publication January 20, 2005

Reprint requests should be addressed to Dr. Kazuma Kishi, the Department of Respiratory Medicine, Respiratory Center, Toranomon Hospital, 2-2-2 Toranomon, Minato-ku, Tokyo 105-8470 


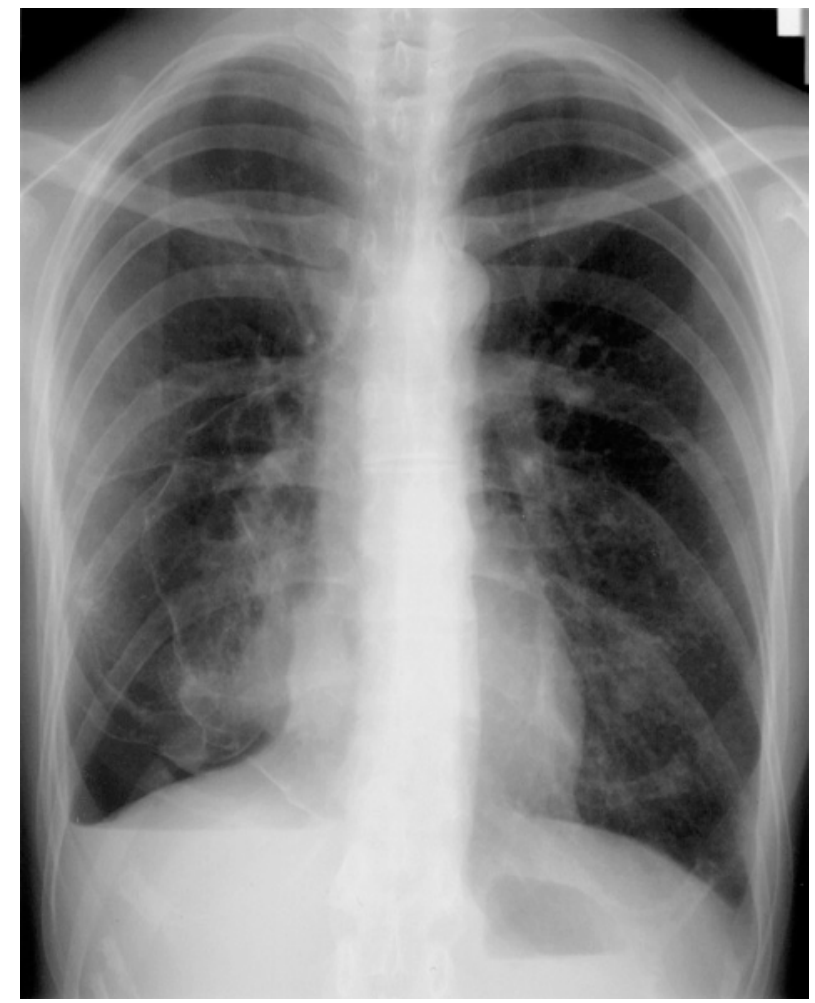

Figure 1. A chest radiograph taken on admission showed a mass in the right lower lung field, and ipsilateral pneumothorax and a small amount of pleural effusion.

was noted in the upper half of the pleural cavity, and thickening of both parietal and visceral pleura was seen in the rest of the lower part of the thoracic cavity. Multiple thin-walled cysts were observed throughout beneath the thickened visceral pleura. There was a small-amount, but non-chylous pleural effusion. Although the discrete mass lesion seen on the chest CT was not precisely identifiable macroscopically, a wedge resection of the slightly bulged region of the superior segment of the right lower lobe was performed.

Histologic examination of the resected specimen revealed a deep folding of the thickened visceral pleura. Lung parenchyma adjacent to the deep folding had collapsed (Fig. 3A). These findings were compatible with rounded atelectasis. In the rounded atelectasis and the remaining resected lung, cystic air spaces and nodular proliferation of abnormal smooth muscle cells distributed into the perivascular and peribronchiolar areas were recognized. Hemosiderin-laden macrophages were present in the alveolar spaces close to the area where proliferation of abnormal small blood vessels was observed (Fig. 3B). There was a ring-like calcification in the vascular walls. Immunohistochemically, smooth muscle cells were stained with a monoclonal antibody against HMB45. Based on these findings, the histopathological diagnosis of rounded atelectasis associated with LAM was made.

The patient has been well since VATS and has been under



Figure 2. A chest CT scan revealed multiple thin-walled cysts, pneumothorax with pleural effusion and a rounded mass containing scattered calcification in contact with thickened pleura.

close observation without any treatment.

\section{Discussion}

Rounded atelectasis is an uncommon parenchymal collapse of the lung that was first described by Loeschke (4) in 1928 in the German literature, and was later documented as the "folding lung" by Blesovsky (5) in English in 1966. Asbestos exposure accounts for the majority of reported cases. Other etiologies included posttherapeutic pneumothorax (6), congestive heart failure (7), postthoracotomy (8), posttraumatic rib fractures with hemorrhagic effusion (1), and legionella pneumonia (9). The duration of developing rounded atelectasis varies from several months to years according to the published reports $(1,9)$. In the present patient, the rounded atelectasis had been formed within 18 months based on the sequential radiographic findings.

There are two hypothetic theories for the pathogenesis of rounded atelectasis. One hypothesis proposed by Hanke and Kretzschmar (6) is that rounded atelectasis develops as a result of accumulation of pleural effusion which then causes the partially aerated portion of the lung to float. A cleft subsequently forms in the collapsed section and that portion of the lung is forced to tilt on itself. As the effusion resolves, fibrinous adhesions bind the tilted portion of the lung in place resulting in an atelectatic parenchymal mass. The second hypothesis advocated by Menzies and Fraser (10) is that 

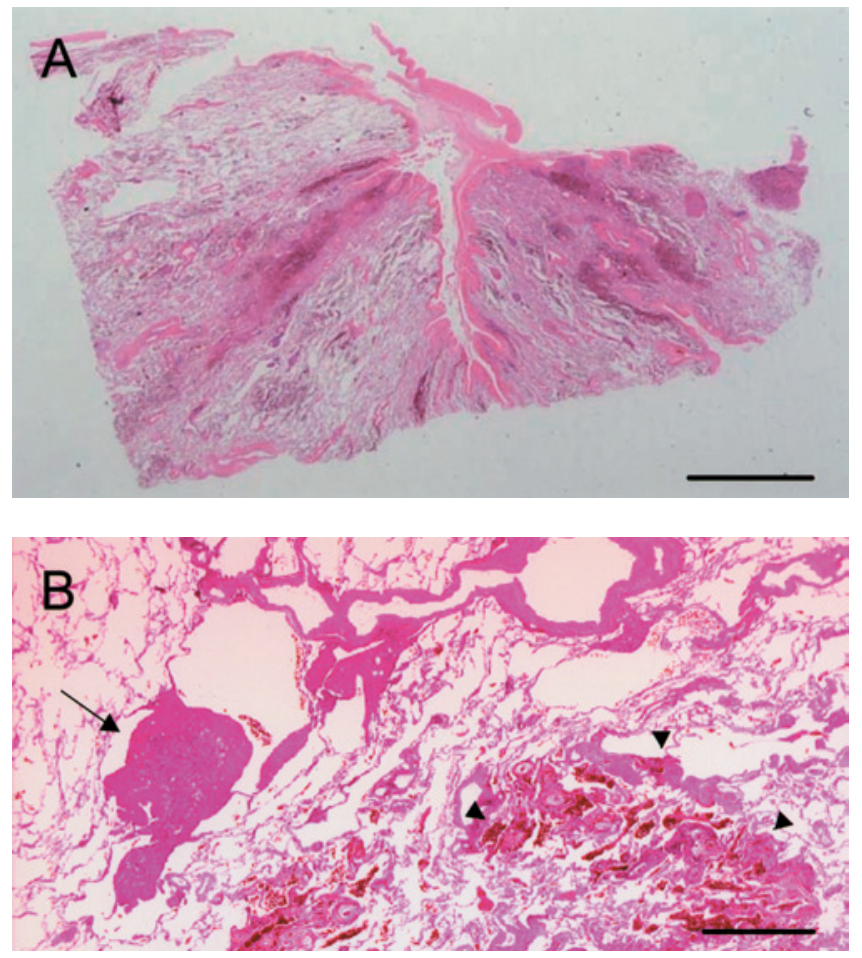

Figure 3. The mass lesion had histopathological characteristics of rounded atelectasis associated with LAM. A: Photomicrograph showing deep folding of the thickened visceral pleura. Lung parenchyma underlying the fibrosis had collapsed (HE stain). The bar indicates $5 \mathrm{~mm}$. B: Nodular proliferation of abnormal smooth muscle cells was seen adjacent to the thinwalled cyst (arrow). Hemosiderin-laden macrophages were observed in the alveolar spaces near small blood vessels (arrowheads) (HE stain). The bar indicates $500 \mu \mathrm{m}$.

pleuritis is the primary event that leads to pleural fibrosis. As the fibrous tissue matures, it contracts and causes eventual folding of the underlying pleura, and that in turn leads to the collapse of the underlying lung parenchyma. Because of extensive pleural adhesion, the second theory might be rather applicable to the mechanism of developing rounded atelectsis in the present case.

LAM can occur sporadically or as a part of tuberous sclerosis. The present patient is considered to be suffering from sporadic LAM because she had no family history of tuberous sclerosis or lacked the clinical features of tuberous sclerosis such as angiofibroma, epilepsy or mental retardation.

To our knowledge after careful review of the written literature, the present case is the first description of rounded atelectasis as a complication of LAM or tuberous sclerosis. Taylor et al (2) reported that $81 \%$ of the patients with LAM developed pneumothorax during their disease course. Although the present patient did not have any episode of pneumothorax or experience chest pain or dyspnea, her chest radiograph on admission demonstrated right-sided pneumothorax. We assume that recurrent and subclinical pneumothoraces have caused inflammation of the pleura, resulting in the development of rounded atelectasis. In fact, multiple pleural adhesions followed by recurrent pneumothoraces have been the most important problems complicating lungtransplantation surgery in patients with LAM (3).

The chest CT findings of rounded atelectasis are well described (11). Rounded atelectasis appears as a rounded masslike opacity adjacent to the thickened pleura and with curvilinear opacities of bronchi and vessels (i.e., the comet tail sign). The radiographic characteristics of the lesion in our patient are consistent with those documented CT features of rounded atelectasis, except for scattered calcification in the mass. The mechanisms for the development of calcification remain undetermined, but it is presumed that calcification might have developed after repeated pulmonary hemorrhage that was caused by pulmonary vascular occlusion due to rounded atelectasis and/or nodular proliferation of abnormal smooth muscle cells in vascular walls.

In summary, we have reported an unusual case of rounded atelectasis associated with LAM characterized by the formation of a mass lesion containing scattered calcification. The conditions described here may help in furthering our understanding of the pathogenesis of rounded atelectasis as well as LAM.

\section{References}

1) Hillerdal G. Rounded atelectasis. Clinical experience with 74 patients. Chest 95: 836-841, 1989.

2) Taylor JR, Ryu J, Colby TV, Raffin TA. Lymphangioleiomyomatosis: clinical course in 32 patients. N Engl J Med 323: 1254-1260, 1990.

3) Boehler A, Speich R, Russi EW, Weder W. Lung transplantation for lymphangioleiomyomatosis. N Engl J Med 335: 1275-1280, 1996.

4) Loeschke H. In: Handbuch der speziellen Anatomie und Histologie. Springer Verlag, Berlin 3 (pt 1): 599, 1928.

5) Blesovsky A. The folded lung. Br J Dis Chest 60: 19-22, 1966.

6) Hanke R, Kretzschmar R. Round atelectasis. Semin Roentgenol $\mathbf{1 5}$ 174-182, 1980.

7) Stark P. Round atelectasis: another pulmnary pseudotumor. Am Rev Respir Dis 125: 248-250, 1982.

8) Cuasay R, Zehr R. Round atelectasis in an elderly man: the role of thoracotomy. Heart Lung 15: 202-204, 1986.

9) Kuno R, Bower DJ, Aguayo SM. Round atelectasis as a complication of Legionnella pneumonia. Chest 106: 607-608, 1994.

10) Menzies R, Fraser R. Round atelectasis; pathologic and pathogenetic features. Am J Surg Pathol 11: 674-681, 1987.

11) Batra P, Brown K, Hayashi K, Mori M. Rounded atelectasis. J Thorac Imag 11: 187-197, 1996. 